\title{
Traduire
}

Une autre perspective sur r tr traduction

Revue française de la traduction

$237 \mid 2017$

La tête dans la toile

\section{Technologies de rupture sur le web. Les traducteurs (presque) rompus à l'exercice}

\section{Anne-Marie Robert}

\section{(2) OpenEdition}

1 Journals

Édition électronique

URL : http://journals.openedition.org/traduire/947

DOI : $10.4000 /$ traduire.947

ISSN : 2272-9992

Éditeur

Société française des traducteurs

Édition imprimée

Date de publication : 1 décembre 2017

Pagination : 48-56

ISSN : 0395-773X

\section{Référence électronique}

Anne-Marie Robert, «Technologies de rupture sur le web. Les traducteurs (presque) rompus à

l'exercice », Traduire [En ligne], 237 | 2017, mis en ligne le 01 décembre 2017, consulté le 15 novembre 2019. URL : http://journals.openedition.org/traduire/947 ; DOI : 10.4000/traduire.947 


\section{Technologies de rupture sur le web Les traducteurs (presque) rompus à l'exercice}

\section{Anne-Marie Robert}

\section{Introduction}

Le métier de traducteur est en pleine mutation. À tel point que sa désignation évolue vers une nouvelle terminologie faisant référence à son statut, son industrie, son informatisation, son activité, sa spécialisation, son multitâchisme ou son humanité : " ingénieur en communication multilingue et multimédia ", " localisateur ", " post-éditeur ", " coordinateur linguistique ", " transcréateur ", "langagier " ou encore " biotraducteur " (par opposition aux moteurs de prétraduction automatique).

Dans ce contexte, force est de constater que les innovations technologiques ne cessent de révolutionner et de refaçonner les pratiques du traducteur professionnel. À l'ère du toutnumérique et du tout-connecté, le traducteur internaute dispose de technologies et d'outils (smartphones, tablettes, ordinateurs portables, ordinateurs de bureau) qu'il intègre/qui s'intègrent à son environnement de travail.

Sur le web, les technologies de rupture ont commencé à bouleverser différentes sphères de la société et à transformer le travail. L'internet mobile, les objets connectés, l'informatique dématérialisée, les mégadonnées et la traduction collaborative ont des conséquences sur le lieu de travail, le rapport au travail et le bien-être au travail du traducteur. Ces mutations technologiques passent par des phases d'information, de formation, d'adaptation et d'appropriation.

\section{Interaction entre les technologies de rupture et le traducteur}

\subsection{Internet mobile et nomadisation}

Grâce à la connexion internet sur son téléphone mobile, le traducteur est en mesure de travailler différemment. L'internet mobile favorise la connectivité, la réactivité et l'instantanéité en tout temps et en tout lieu et ses applications sont nombreuses dans l'univers du traducteur. 
Ce dernier dispose d'un accès direct à sa messagerie électronique et peut ainsi gérer ses courriels en temps réel, répondre à ses clients presque instantanément, émettre un devis avec une réactivité extrême, et ce quel que soit l'endroit où il se trouve.

Il accède à des applis métiers qui facilitent les tâches administratives (gestion et suivi de son compte bancaire, de ses déclarations fiscales, de ses contrats d'assurance, de ses abonnements professionnels), la gestion de ses déplacements professionnels (géolocalisation et guidage pour se rendre à un rendez-vous d'affaires, achat et échange de m-billets de transport, suivi de ses trajets/voyages/correspondances en temps réel, notification de perturbations dans les transports en commun, réservation d'hôtels), la communication (visioconférence y compris à l'international, presse et stations de radio du monde entier, multimédia pour visionner une démo de produit), l'organisation (agenda, gestion d'événementiel pour s'inscrire à un colloque, planification pour indiquer ses disponibilités) ou encore applis pratiques (enregistrement des coordonnées d'un contact professionnel dont la carte de visite possède un flashcode, commande de consommables en ligne, numérisation et conversion au format PDF d'un document papier).

II navigue sur internet pour vérifier une information ou encore consulter les fils d'actualité auxquels il est abonné sur Facebook, Twitter ou Linkedln et même y contribuer en direct depuis le quai d'un métro, par exemple.

\subsection{Objets connectés et capteurisation}

Le marché des objets connectés (Internet of Things en anglais) en tous genres explose et de nouvelles sources d'informations voient le jour. Un objet connecté implique une double technologie matérielle et logicielle, avec un objet physique comportant des capteurs qui communiquent avec une appli installée sur un smartphone. Le traducteur peut s'appuyer sur des objets connectés pour veiller à son bien-être, sa qualité de vie et sa santé au travail.

Il peut se simplifier la vie en utilisant un assistant automatisé à commande vocale pour l'aider à gérer son planning et ses tâches quotidiennes sous forme de rappels, contrôler l'éclairage et la température de son bureau ou encore lancer des recherches sur internet.

Il peut mesurer la qualité de l'air de son lieu de travail et l'assainir avec un purificateur d'air connecté filtrant les polluants, allergènes, bactéries, virus et odeurs. II peut gérer le taux de luminosité et réduire ou augmenter l'opacité d'une fenêtre en lui appliquant un film plastique intelligent. II peut s'équiper d'un atténuateur de bruit ambiant connecté pour l'aider à rester concentré.

II peut surveiller sa santé en portant une montre connectée qui mesure sa fréquence cardiaque, son taux d'oxygène dans le sang et le nombre de pas qu'il effectue. II peut prévenir tout stress trop important, voire un burn-out, grâce à des objets connectés qui analysent la 
tonalité de sa voix, la qualité de son sommeil ou le nombre de fautes dans ses courriels, par exemple. II peut surveiller l'ergonomie de son poste de travail pour prévenir les troubles musculo-squelettiques : bureau intelligent qui s'adapte à la corpulence et à la physionomie de l'utilisateur pour optimiser son confort, chaise de bureau connectée qui vibre en cas de mauvaise posture ou de position assise prolongée ou encore patches connectés pour soulager ses douleurs lombaires.

\subsection{Nuage et dématérialisation}

Le nuage (Cloud en anglais) virtualise l'environnement de travail du traducteur. Les ressources informatiques dématérialisées sont accessibles uniquement sur internet, qu'il s'agisse de logiciels, de solutions, de services, de plates-formes ou d'infrastructures. Cet environnement est de ce fait en train de se transformer, de se simplifier et de s'alléger pour gagner en convergence, en puissance, en souplesse et en convivialité. Le traducteur peut ainsi accéder à son environnement de travail dématérialisé en temps réel, à partir de tout ordinateur connecté à internet dans le monde entier.

L'informatique dématérialisée crée de nouveaux modèles et entraîne de nouvelles pratiques professionnelles chez le traducteur : nouveau modèle de distribution en ligne de ressources dynamiques, nouveau modèle économique de location en ligne avec tarification à la demande ou à l'utilisation (à l'heure, au mois, à l'année, au giga-octet consommé, au volume de mots ou encore à l'utilisateur), nouveau modèle de gestion informatique automatisée et transparente et nouveau modèle de travail collaboratif (partage de mémoires de traduction, mise à jour dynamique de bases de données terminologiques partagées, messagerie instantanée intégrée à des logiciels dédiés, etc.)(1).

\subsection{Mégadonnées et datafication}

Les données en tous genres s'accumulent de façon exponentielle sur le web. L'exploration et l'analyse de ces mégadonnées (Big Data en anglais) deviennent un enjeu stratégique majeur.

Le traducteur dispose aujourd'hui d'une quantité phénoménale de données exploitables dans l'exercice de sa profession, à condition de maîtriser parfaitement non seulement ses recherches sur internet à l'aide d'outils appropriés (moteurs, métamoteurs, logiciels, opérateurs), mais aussi la gestion de ces données et leur exploration ; que ce soit pour se renseigner sur ses clients et des prospects, pour lancer des recherches thématiques,

(1) Voir l'article «Informatique dématérialisée et logiciels-services : virtualisation de l'environnement de travail du traducteur " référencé dans la bibliographie. 
encyclopédiques, documentaires et terminologiques, pour analyser des données et en dégager des tendances ou encore pour établir des relations/corrélations entre des données et prendre des décisions en connaissance de cause. La disponibilité massive sur internet de corpus multilingues, donc de textes traduits, permet à un traducteur de mettre en place des processus pour optimiser son travail. II peut ainsi décider d'utiliser la technologie d'alignement pour mettre en correspondance des données bilingues et créer sa propre mégamémoire de traduction en amont d'un projet. Cette stratégie dépend de la quantité, de la qualité, de l'actualité et de la pertinence des ressources multilingues disponibles sur internet.

Cet amas d'informations, ces mégadonnées, présentent des spécificités. La gestion de l'information consiste aujourd'hui à gérer non plus des stocks mais des flux de données, qui circulent en permanence et sont immédiatement disponibles et accessibles sur internet. Dans ce contexte, le traducteur devient producteur, transmetteur et consommateur d'informations.

À l'âge de l'information, les données viennent renforcer l'automatisation et les mégadonnées amplifier le développement d'intelligences artificielles. La génération automatique de contenu vient par conséquent rajouter des données aux mégadonnées existantes. Dans le cadre de l'apprentissage automatique, les algorithmes n'ont de cesse d'analyser les informations issues de diverses bases et banques de données (corpus, mémoires de traduction, bases terminologiques multilingues, par exemple) pour enrichir des réseaux neuronaux. Le trait d'union s'effectue naturellement avec la nouvelle technologie neuronale révolutionnaire et... avec la dernière génération de moteurs de traduction automatique neuronale. Cette intelligence artificielle utilise en effet un grand réseau neuronal qui fonctionne sur le modèle du cerveau humain et propose des prétraductions de meilleure qualité à post-éditer.

\subsection{Traduction collaborative et intermédiation}

La traduction collaborative ou participative (Crowdsourcing en anglais) englobe plusieurs réalités. II peut s'agir d'une collaboration entre traducteurs (voir Traduire 233, " Voyage en équipage "). Il peut s'agir des réseaux sociaux qui continuent à révolutionner le positionnement et l'activité commerciale du traducteur sur le web, pour optimiser son e-visibilité à l'international et se faire connaître, pour développer son activité et saisir des opportunités, pour entretenir de manière très pertinente son employabilité ou encore pour socialiser et réseauter virtuellement dans un contexte d'affaires. II peut s'agir de la traduction bénévole de documents par des amateurs au service d'une cause ou d'une communauté (fansubbing d'un jeu vidéo dont la traduction n'est pas prévue dans une langue, par exemple).

Elle peut également prendre la forme d'une mise en relation de traducteurs prestataires de services et de clients via des plates-formes en ligne qui jouent le rôle d'intermédiaires. Ces plates-formes proposent de nouveaux modèles économiques aux pratiques hétéroclites: inscription gratuite, abonnement payant pour le prestataire et gratuit pour le client, abonnement 
payant pour le client et gratuit pour le prestataire, tarification unique en fonction du niveau de qualité souhaité par le client (tarification "standard " ou " business "), comparateur d'offres, présentation sous la forme d'un moteur de recherche mettant automatiquement en relation des clients et des prestataires, solution globale automatisée avec interface de gestion personnalisée, dématérialisation et virtualisation du processus complet (de la commande au paiement), commande en ligne immédiate sans contrat ni négociation, système de recommandation/notation des prestataires par les clients, mise en œuvre du concept de surtraitance ou encore passage d'une économie collaborative à l'ubérisation.

\section{Conséquence des technologies de rupture sur l'environnement, le rapport et le bien-être au travail du traducteur}

L'impact de ces technologies de rupture sur le traducteur et ses pratiques prend différentes formes qui comportent des avantages comme des inconvénients.

\subsection{Puissance des médias sociaux}

Avec les médias sociaux, le traducteur devient un média en puissance qui dispose d'un espace d'expression inégalé. Sur le web, la parole est multicanale (articles, photos, vidéos, musique, etc.). Le traducteur a autant de place et de moyens que les institutionnels, les multinationales ou la presse pour communiquer, revendiquer et vendre ses prestations de services en traduction. Cela modifie son rapport aux autres : il détient des informations sur ses clients, ses confrères et les différentes parties prenantes de sa profession. Du fait de cette surexposition, le traducteur doit veiller à son e-réputation. Sur le web, la parole est sans grade statutaire et les traducteurs développent des échanges horizontaux, avec un sentiment égalitaire et une vision différente de la hiérarchie et du collectif. Le web défie toutes les lois de la diffusion. Tout message peut être diffusé facilement et gratuitement via de multiples canaux, avec une puissance et une résonance décuplées à l'échelle internationale pour les traducteurs. Le web est communautaire. Les traducteurs se regroupent par centre d'intérêt.

Tous ces éléments transforment la nature du travail (qui relève désormais essentiellement du traitement de l'information) et celle du pouvoir.

\subsection{Socialisation en ligne}

Avec tous les outils de communication sur internet, le traducteur souffre moins de solitude et d'isolement. Le réseautage, même virtuel, lui permet de sortir de sa bulle et de socialiser en ligne. 


\subsection{Gain en autonomie}

Grâce à internet, le traducteur gagne en indépendance. II a accès à d'innombrables ressources, peut faire de la veille et développer ses propres relations et collaborations. II doit toutefois veiller à résister à la tentation de la flânerie numérique et de l'errance sans fin sur les réseaux aux dépens de la productivité et de l'efficacité.

\subsection{Porosité entre vie professionnelle et vie personnelle}

Avec internet, la vie privée et la vie professionnelle du traducteur peuvent coexister et se confondre : le travail ne fonctionne plus en vase clos. Le traducteur peut le vivre comme une bouffée d'oxygène, un décloisonnement ou comme une intrusion du travail à la maison et vice versa en raison de l'ultraconnectivité. Dans ce contexte, le droit à la déconnexion et le devoir de déconnexion deviennent de nouveaux enjeux dans l'équilibre entre vie privée et vie professionnelle.

\subsection{Risques psychosociaux}

L'usage d'internet peut induire des risques psychosociaux, donc d'exposition au stress professionnel chez les traducteurs. Ces risques peuvent découler du sentiment de sursollicitation, de surcharge et d'incapacité à aller au bout des choses dans un contexte de multitâchisme effréné ; du sentiment de saturation mentale du fait des flux et reflux sans fin d'informations (l'information motive autant qu'elle épuise, fascine autant qu'elle agace, libère autant qu'elle aliène) ; du sentiment d'intrusion dû à la traçabilité par l'omniprésence de capteurs (géolocalisation, données personnelles issues d'objets connectés) pouvant déboucher sur le syndrome Big Brother: sentiment de surveillance de ses données et de traçabilité de ses actions, intériorisation du regard des autres sur sa façon de vivre, de penser et d'agir ou encore impression de traque de ses comportements.

\subsection{Risques d'ubérisation}

Les plates-formes en ligne présentent le risque d'ubérisation de la profession de traducteur. Au-delà des nouveaux modèles économiques proposés par ces plates-formes, leurs pratiques sur le web posent de nombreuses questions déontologiques : tarifs imposés sans négociation, tarifs uniques sans analyse du besoin de la prestation et du travail nécessaire pour y répondre, problème du lien de subordination, concurrence déloyale sans vérification de l'exercice légal de la profession par le prestataire, déshumanisation de la prestation de services du fait d'une automatisation totale de ces plates-formes, incitation à importer ses mémoires de traduction personnelles comme "preuve de sérieux ", etc. 
Partant du principe que la qualité de vie au travail est un levier de performances, la bonne santé et le bon rapport au travail sont des facteurs essentiels pour permettre au traducteur de mener à bien ses prestations de services intellectuelles dans un environnement de travail épanouissant. II doit donc savoir se ménager, prendre soin de lui, veiller à son propre équilibre physique et psychique et se ressourcer.

\section{3. Éléments de réflexion pour s'approprier les mutations technolo- giques sur le web}

\subsection{Vers de nouveaux modes d'auto-apprentissage des connaissances de base}

Dans le domaine des connaissances générales, il serait de moins en moins utile d'apprendre puisque toutes les informations de base sont accessibles sur internet. Étant donné la disponibilité massive d'informations, chacun peut maintenant acquérir des connaissances sans l'aide d'un enseignant. Serait-ce la fin programmée des cours généraux en formation initiale ? La généralisation à venir de la pédagogie inversée ? L'explosion de l'auto-apprentissage avec notamment les cours en ligne ouverts à tous (MOOC en anglais) ?

\subsection{Vers un développement des capacités cognitives pour mieux appréhender le traitement de l'information}

Avec internet, un nouveau rapport au savoir est en train d'émerger, nécessitant d'apprendre à traiter l'information de manière économique en limitant la charge mentale mobilisée à cet effet. La méthodologie cognitive et l'évaluation de la fiabilité des données devraient devenir des disciplines incontournables. En d'autres termes, savoir collecter, extraire, traiter, trier, filtrer et évaluer l'information qui nous submerge en permanence.

\subsection{Vers un accompagnement pour mieux vivre en réseaux virtuels et travailler, voire télétravailler, de manière indépendante}

En donnant plus de place au virtuel, le rapport au travail des jeunes générations évolue, les poussant à rechercher des statuts indépendants cohérents avec leurs techniques de production qui fonctionnent dans des logiques de réseaux. L'explosion des nouvelles technologies a d'ailleurs accentué le phénomène de l'entrepreneuriat en télétravail.

\subsection{Vers l'introduction de modules de formation spécifiques intégrant les mutations technologiques pour optimiser l'employabilité du traducteur}

En formation initiale comme en formation continue, les programmes devraient intégrer les thématiques suivantes : applis mobiles professionnelles, objets connectés, outils dématérialisés, 
optimisation des recherches sur internet, gestion des mégadonnées pour enrichir ses ressources grâce à la technologie de l'alignement, plates-formes collaboratives en ligne avec leurs points de vigilance, gestion des médias et réseaux sociaux, installation en tant que traducteur indépendant.

\subsection{Vers une remise en cause régulière de ses schémas mentaux}

II conviendrait de réapprendre régulièrement à penser différemment pour s'adapter. Intégrer de nouvelles façons de penser pour renforcer ses capacités d'adaptation implique de sortir de ses zones de confort, de faire preuve d'ouverture d'esprit voire de se faire violence pour changer ses habitudes.

\section{Conclusion et prospective}

La convergence entre les biotraducteurs et les technologies de rupture passe par la formation pour accompagner technologiquement, psychologiquement et stratégiquement le traducteur à faire face aux mutations technologiques et à leurs conséquences sur les pratiques professionnelles. Et faire ainsi en sorte que le travail procure davantage de bien-être que de stress au traducteur.

Du point de vue de la prospective, la prochaine étape sera vraisemblablement la mutation des intelligences artificielles en cobotique (robotique collaborative) qui ira au-delà d'une simple interaction ou même collaboration homme-machine, et introduira une véritable coopération homme-machine, opera signifiant " faire œuvre commune ".

tilt.communications@wanadoo.fr

Anne-Marie Robert est titulaire d'un Master de traduction professionnelle (ITIRI, Université de Strasbourg). Elle exerce en tant que traductrice technique, informatique et marketing indépendante depuis 1997, de l'anglais et l'espagnol vers le français. Devenue formatrice-consultante dans ses domaines de spécialité (nouvelles technologies, TAO, post-édition, localisation informatique et multimédia, communication technico-marketing), elle enseigne dans plusieurs masters de traduction en France et participe en tant qu'experte à des projets de l'Union européenne. Elle est également activement engagée au sein d'institutions professionnelles : la SFT (Société française des traducteurs), I'UNAPL (Union nationale des professions libérales) et le CESER Auvergne-Rhône-Alpes (Conseil économique, social et environnemental régional). 


\section{Bibliographie}

MASSARDO Isabella et VAN DER MEER Jaap, 2017, The Translation Industry in 2022 - Report from the TAUS Industry Summit - Amsterdam, March 22-24, 2017, De Rijp (Pays-Bas), TAUS BV.

METTLING Bruno, 2015, Transformation numérique et vie au travail, Paris, Ministère du Travail, de l'Emploi, de la Formation Professionnelle et du Dialogue Social.

ROBERT Anne-Marie, 2011, "Informatique dématérialisée et logiciels-services : virtualisation de l'environnement de travail du traducteur ", in Traduire, 224, p. 14-20. 